\title{
Parallel Array Bistable Stochastic Resonance System with Independent Input and Its Signal-to-Noise Ratio Improvement
}

\author{
Wei Li, Hanzhi Lu, and Yanyan Zuo \\ Harbin Institute of Technology Shenzhen Graduate School, Shenzhen 518055, China \\ Correspondence should be addressed to Wei Li; li.wei@hitsz.edu.cn
}

Received 6 March 2014; Revised 10 July 2014; Accepted 12 July 2014; Published 27 August 2014

Academic Editor: Wuquan Li

Copyright ( 2014 Wei Li et al. This is an open access article distributed under the Creative Commons Attribution License, which permits unrestricted use, distribution, and reproduction in any medium, provided the original work is properly cited.

\begin{abstract}
We study the design enhancement of the bistable stochastic resonance (SR) performance on sinusoidal signal and Gaussian white noise. The bistable system is known to show an SR property; however the performance improvement is limited. Our work presents two main contributions: first, we proposed a parallel array bistable system with independent components and averaged output; second, we give a deduction of the output signal-to-noise ratio (SNR) for this system to show the performance. Our examples show the enhancement of the system and how different parameters influence the performance of the proposed parallel array.
\end{abstract}

\section{Introduction}

Stochastic resonance has attracted considerable attention over the past decades. SR is defined as a phenomenon that is manifest in nonlinear systems whereby generally feeble input information (such as a weak signal) can be amplified and optimized by the assistance of noise.

The physical mechanism of SR has been known since the initial work by Benzi et al. at the beginning of the 1980s [1-3] and received much attention by the physical community in the following years. SR has been observed in a large variety of systems, including bistable ring lasers and semiconductor devices. The first discussed and most developed SR mechanism was the bistable system. Since it has a precise mathematical expression and can be interpreted visually, the bistable system draws much attention of the researchers.

SR can be envisioned as a particular problem of signal extraction from background noise. It is quite natural that a number of authors tried to characterize SR within the formalism of data analysis, most notably by introducing the notion of SNR [4-6]. The focus of our present work is on bistable system and its SNR improvement. SNR is a very important quantity, since it influences information, detection, estimation, and many other measures [7].
The early study of SR system focused on nature nonlinear system to analyse its properties [1-3]. Later, the benefit of the system was known, and researchers started to design new systems to meet the need in engineering to enhance the performance of the system. Many works dealt with SR in engineering such as signal estimation and detection [810]. A good way of designing the SR system is using array since array can enhance system performance which is widely studied [11-14]. The array for SR systems can be designed either in coupled way $[12,15-18]$ or in uncoupled way $[13,14]$. For the coupled array, the processing in each component is complicated due to coupling with other components. Uncoupled parallel array has been widely studied in SR system due to its simplicity, such as superthreshold system. For bistable system, the work in [19] gives a brief introduction of a type of array enhancement for the sinusoidal signal in bistable array with a similar structure as superthreshold system. In [20] the theory for this type of array is demonstrated. However, even with the uncoupled components, the performance of the system still has room to be improved. Since, in these types of array, the components are not independent of each other, the independence in statistics is an importance feature to the best performance.

This paper is in fact inspired by traditional parallel system, proposes a new parallel array with independent 
sensors, and focuses on the output SNR performance. This is different from traditional parallel SR system since traditional system uses one receiving sensor and parallel array processing components so that input for each component is not independent in statistics. And it is also different from traditional array signal processing [7] since we do not need to consider the shape of the array. To simplify the analysis, we limit our study to two-state bistable system driven by sinusoidal signal and Gaussian white noise and assume some identical independent settings in every bistable component. To analyse the performance of this array theoretically, we give a complete proof on output SNR and experiments to demonstrate the parameter influences.

This paper is organized as follows. The framework of two-state model of bistable system is described in Section 2. Section 3 deals with the case that a new structure of the parallel array is assigned to bistable system and the output SNR of this system is deduced. Section 4 is devoted to instances of the proposed system whose performance is indeed enhanced by adding noise. And the influence of the parameter on the system is also analysed in this section. Finally, in Section 5, we summarize the following.

Notation. $E(\cdot)$ stands for ensemble average, upper dot $\dot{a}$ denotes a time derivative of $a, A^{\prime}(b)$ represents the derivative of $A$ with respect to $b, \delta(\cdot)$ is Dirac delta function, and $f * g$ represents the convolution of $f$ and $g$.

\section{Two-State Model of Bistable Systems}

We consider the overdamped motion of a Brownian particle in a bistable potential in the presence of noise and periodic forcing $[21,22]$. The system can be presented by FokkerPlanck equation. Consider

$$
\dot{x}(t)=-U^{\prime}(x)+A_{0} \cos (\Omega t)+\xi(t),
$$

where $x$ is the position of Brownian particle, $U(x)$ denotes the reflection-symmetric quartic potential,

$$
U(x)=-\frac{a}{2} x^{2}+\frac{b}{4} x^{4}
$$

$\xi(t)$ denotes a zero-mean, Gaussian white noise with variance $2 D$, and $A_{0} \cos (\Omega t)$ is periodic forcing. The potential $U(x)$ is bistable with minima located at $\pm x_{m}$, with $x_{m}=(a / b)^{1 / 2}$. The height of the potential barrier between the minima is given by $\Delta U=a^{2} / 4 b$.

To simplify the problem in this paper we discuss twostate model $[23,24]$ that epitomizes the class of symmetric bistable systems introduced. Such a discrete model under certain restrictions renders an accurate representation of most continuous bistable systems. Let us consider a symmetric unperturbed system that switches between two discrete states $\pm m_{x}$. We define $n_{ \pm}(t)$ to be the probabilities that the system occupies either state \pm at time $t$; that is, $x(t)= \pm x_{m}$. Then

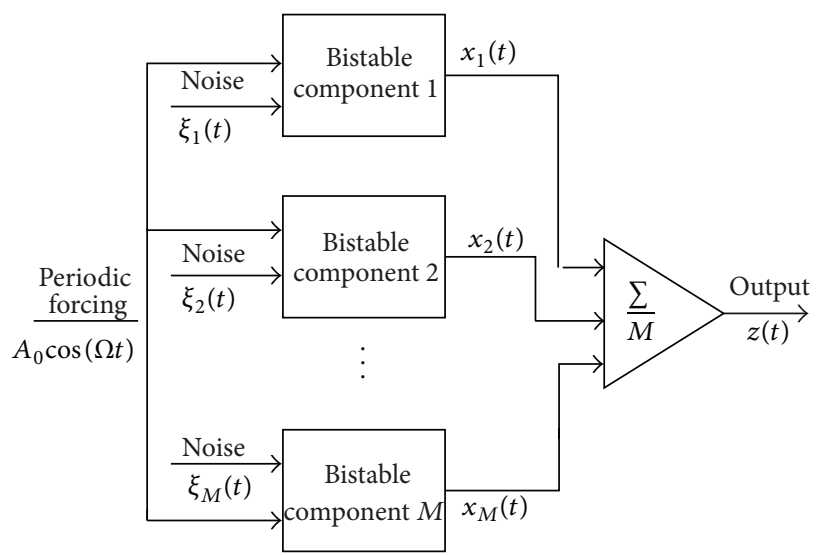

FIgure 1: The parallel bistable array with $M$ independent components.

the power spectral density of this symmetric bistable system commonly reported in the literature [21] is

$$
\begin{aligned}
S(\omega)= & {\left[1-\frac{1}{2}\left(\frac{A_{0} x_{m}}{D}\right)^{2} \frac{4 r_{k}^{2}}{4 r_{k}^{2}+\Omega^{2}}\right] \times \frac{4 r_{k} x_{m}^{2}}{4 r_{k}^{2}+\omega^{2}} } \\
& +\frac{\pi}{2}\left(\frac{A_{0} x_{m}}{D}\right)^{2} \times \frac{4 r_{k} x_{m}^{2}}{4 r_{k}^{2}+\Omega^{2}}[\delta(\omega-\Omega)+\delta(\omega+\Omega)],
\end{aligned}
$$

in which Kramers rate

$$
r_{k}=\frac{1}{\sqrt{2} \pi} \exp \left(-\frac{\Delta U}{D}\right) .
$$

It is rate of transitions between the neighboring potential wells caused by the fluctuational forces.

Since the noise in the output of the system is no longer Gaussian, the definition $\operatorname{mean}^{2} /$ variance for SNR is not suitable in this system. Here we adopt the definition for input and output SNR according to [21] as follow:

$$
\mathrm{SNR}=\frac{2\left[\lim _{\Delta \omega \rightarrow 0} \int_{\Omega-\Delta \omega}^{\Omega+\Delta \omega} S(\omega) d \omega\right]}{S_{N}(\Omega)} .
$$

For the weak signal $\left(A_{0} x_{m} \ll \Delta U\right)$, we can omit high order items; then the output SNR for this symmetric bistable system is approximately

$$
\mathrm{SNR}=\pi\left(\frac{A_{0} x_{m}}{D}\right)^{2} r_{k}
$$

\section{Parallel Bistable Array with Independent Components}

3.1. Proposed Array and Its Output SNR. In this section, we discuss the parallel array bistable system and its SR performance.

We consider the parallel array with $M$ components in Figure 1. Each component has a receiving sensor and a 
processing property as described in the last section. We suppose that the receiving time difference $\Delta t \ll T_{\Omega}=2 \pi / \Omega$ but there still is a considerable distance between every two different components. This can be set by a suitable $\Omega$. Then the input periodic signal can be taken as $A_{0} \cos (\Omega t)$ for all the components. And the noise $\xi_{i}(t)$ is independent and identically distributed (IID) for each input, and the output is $x_{i}(t)$ for the $i$ th component; then all the outputs are averaged and the response of the array is given as

$$
z(t)=\frac{\sum_{i=1}^{M} x_{i}(t)}{M}
$$

In the following of this section, we present the main results with respect to the parallel array bistable system. Two theorems form the SR performance analysis on output SNR. We utilize four lemmas for proving the theorems. The proofs of all the theorems and lemmas of this section are relegated to appendices.

Theorem 1. For the parallel bistable system with $M$ components, the output SNR is

$\operatorname{SNR}(M)$

$$
=\frac{\pi\left(A_{0} x_{m} / D\right)^{2} r_{k}}{-(1 / 2)\left(A_{0} x_{m} / D\right)^{2}\left(4 r_{k}^{2} /\left(4 r_{k}^{2}+\Omega^{2}\right)\right)-H(M)},
$$

where

$$
H(M)= \begin{cases}F(M), & \text { if } M \text { is even } \\ G(M), & \text { if } M \text { is odd, }\end{cases}
$$

in which

$$
\begin{aligned}
& F(M)=\left(\frac{1}{2}\right) \sum_{k=0}^{M / 2-1} \frac{2 k-M}{M}\left(\begin{array}{c}
M \\
k
\end{array}\right) \\
& \times\left\{\sum_{i=0}^{k} \sum_{j=0}^{M-k}\left(\begin{array}{c}
k \\
i
\end{array}\right)\left(\begin{array}{c}
M-k \\
j
\end{array}\right) B^{M-i-j} f(M-i-j)\right. \\
& G(M)=\left(\frac{1}{2}\right)^{M(M-1) / 2} 2 k-M\left(\begin{array}{c}
M \\
\left.\sum_{k=0}^{M-k-j}+(-1)^{k-i}\right]
\end{array}\right\} \\
& \times\left\{\sum_{i=0}^{M} \sum_{j=0}^{M-k}\left(\begin{array}{c}
k \\
i
\end{array}\right)\left(\begin{array}{c}
M-k \\
j
\end{array}\right) B^{M-i-j} f(M-i-j)\right. \\
& \times\left[\begin{array}{c}
\left.(-1)^{M-i-j}+(-1)^{k-i}\right] \\
k
\end{array}\right\}
\end{aligned}
$$

In the above equations,

$$
\begin{gathered}
B=\frac{2 r_{k}\left(A_{0} x_{m} / D\right)}{\sqrt{\left(4 r_{k}^{2}+\Omega^{2}\right)}}, \\
f(x)=\frac{\Gamma((x+1) / 2)}{\sqrt{\pi} \Gamma(x / 2+1)},
\end{gathered}
$$

and $\Gamma$ is gamma function and defined as

$$
\Gamma(x)=\int_{0}^{\infty} t^{x-1} e^{-t} d t
$$

The theory is based on the following lemmas.

Lemma 2. The $p d f$ of $z(t)$ is

$$
\begin{aligned}
p_{z} & \left(z, t \mid z_{0}, t_{0}\right) \\
& =\sum_{m=0}^{M}\left(\begin{array}{l}
M \\
m
\end{array}\right) n_{+}^{m}(t) n_{-}^{M-m}(t) \delta\left(z-\frac{2 m-M}{M} x_{m}\right) .
\end{aligned}
$$

Lemma 3. If $M$ is even, the autocorrelation function of $z(t)$ is

$$
R_{z}(t+\tau, t)
$$

$$
\begin{aligned}
=x_{m}^{2}\{ & {\left[-\exp \left(-2 r_{k}|\tau|\right) \kappa^{2}(t)+\kappa(t+\tau)\right] \kappa(t) } \\
& -\left(\frac{1}{2}\right)^{M} \exp \left(-2 r_{k}|\tau|\right) \\
& \times \sum_{k=0}^{M / 2-1} \frac{2 k-M}{M}\left(\begin{array}{c}
M \\
k
\end{array}\right) \\
& \times \sum_{i=0}^{k} \sum_{j=0}^{M-k}\left(\begin{array}{c}
k \\
i
\end{array}\right)\left(\begin{array}{c}
M-k \\
j
\end{array}\right) \times B^{M-i-j} \\
& \times \cos (\Omega t-\varphi)^{M-i-j} \\
& \left.\times\left[(-1)^{M-k-j}+(-1)^{k-i}\right]\right\}
\end{aligned}
$$

where

$$
\kappa(t)=B \cos (\Omega t-\varphi) .
$$


Lemma 4. If $M$ is odd, the autocorrelation function of $z(t)$ is

$$
\begin{aligned}
R_{z}(t+\tau, t) & \\
=x_{m}^{2}\{[ & \left.-\exp \left(-2 r_{k}|\tau|\right) \kappa^{2}(t)+\kappa(t+\tau)\right] \kappa(t) \\
& -\left(\frac{1}{2}\right)^{M} \exp \left(-2 r_{k}|\tau|\right) \\
& \times \sum_{k=0}^{(M-1) / 2} \frac{2 k-M}{M}\left(\begin{array}{c}
M \\
k
\end{array}\right) \\
& \times \sum_{i=0}^{k} \sum_{j=0}^{M-k}\left(\begin{array}{c}
k \\
i
\end{array}\right)\left(\begin{array}{c}
M-k \\
j
\end{array}\right) \times B^{M-i-j} \\
& \times \cos (\Omega t-\varphi)^{M-i-j} \\
& \left.\times\left[(-1)^{M-i-j}+(-1)^{k-i}\right]\right\} .
\end{aligned}
$$

Lemma 5. The power spectral density of the output of the parallel array bistable system with $M$ components is

$$
\begin{aligned}
S(\omega)= & -\left[\frac{1}{2}\left(\frac{A_{0} x_{m}}{D}\right)^{2} \frac{4 r_{k}^{2}}{4 r_{k}^{2}+\Omega^{2}}+H(M)\right] \frac{4 r_{k} x_{m}^{2}}{4 r_{k}^{2}+\omega^{2}} \\
& +\frac{\pi}{2}\left(\frac{A_{0} x_{m}}{D}\right)^{2} \frac{4 r_{k}^{2} x_{m}^{2}}{4 r_{k}^{2}+\omega^{2}} \\
& \times[\delta(\omega-\Omega)+\delta(\omega+\Omega)] .
\end{aligned}
$$

Theorem 6. For the weak signal $\left(A_{0} x_{m} \ll \Delta U\right)$, the output SNR for this parallel bistable system with two components is approximately

$$
S N R=2 \pi\left(\frac{A_{0} x_{m}}{D}\right)^{2} r_{k}
$$

3.2. Remark. We conclude this section with three remarks. Our first remark is about the simplified noise. The noise $\xi_{i}(t)$ in practical problems is the sum of two parts in each component. The first part is the receiving noise buried in the receiving signal, and the second part is the tuning noise. Here we suppose that the receiving noise and the tuning noise are independent Gaussian white noise. And the variance of the tuning noise can be set by us. Then we can simplify the noise in each component as Gaussian white noise with $2 D$ variance.

Our second remark is to point out that the proposed array is different from the traditional SR array [20] due to its independent sensors, and it is also different from traditional array signal processing [7] since the shape of the array does not affect the performance. We not only proposed an $M$-array system for bistable SR, but also provided a rigorous proof for the output SNR which is nontrivial as evidenced. And the results also divide $M$ into odd and even situations.

Our third remark is that Theorem 1 can arrive to (6) by setting $M=1$ and Theorem 6 by setting $M=2$. In

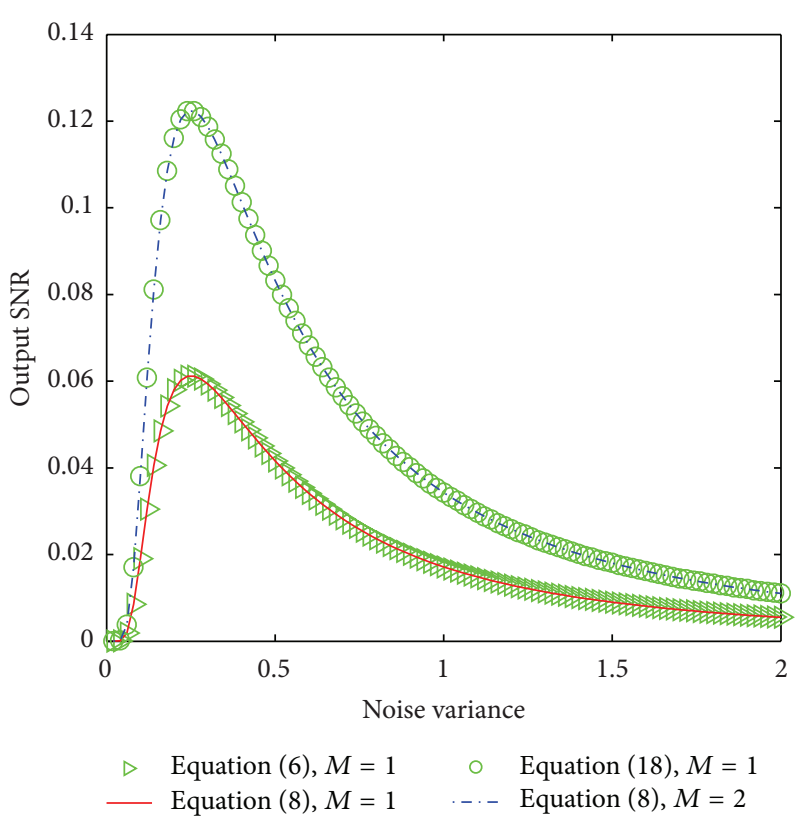

FIGURE 2: Output SNR as a function of noise variance with $A_{0}=0.1$, $a=1, b=1$, and $f=100$.

fact, Figure 1 shows that if $M=1$, the system without array becomes the conventional single bistable SR system. The equivalence is also shown in Figure 2 on the same other parameters.

\section{Simulation Results}

We now provide examples to illustrate the properties of our proposed bistable parallel array system.

4.1. SR Effect and the Influence of $M$. For illustration of the possibility of an SR in the output SNR, we consider two different systems based on the theory of (8) for the proposed array: case A: $a=1, b=1, A=0.1$, and $f=100$ in Figure 3(a); case B: $a=1.5, b=1, A=1$, and $f=10$ in Figure 3(b). Though the two systems are set by different parameters, they both display evolutions of the resulting output SNR of (8), as a function of the noise variance, in some typical conditions. Since $D$ cannot be zero in (8), all the curves start from a small amount $D$ close to zero. As noise power increases, the SR peak rises, shifts to higher noises, and then subsides. This result shows us that if the input noise variance is smaller than the peak point, the tuning noise can be added to improve the output SNR performance. For increasing $M$, the efficiency of the array and the maximum output SNR increase. This demonstrates that the array of nonlinear devices can play the role of an SNR amplifier, in definite conditions.

At $M=2$, SR effect gets more pronounced than at $M=1$ which is the traditional SR bistable system. The output SNR is twice the output of the traditional bistable system according to Theorem 6. As $M$ increases, SR effect gets more pronounced. However if $M$ is even $(M \geq 2)$, the output 

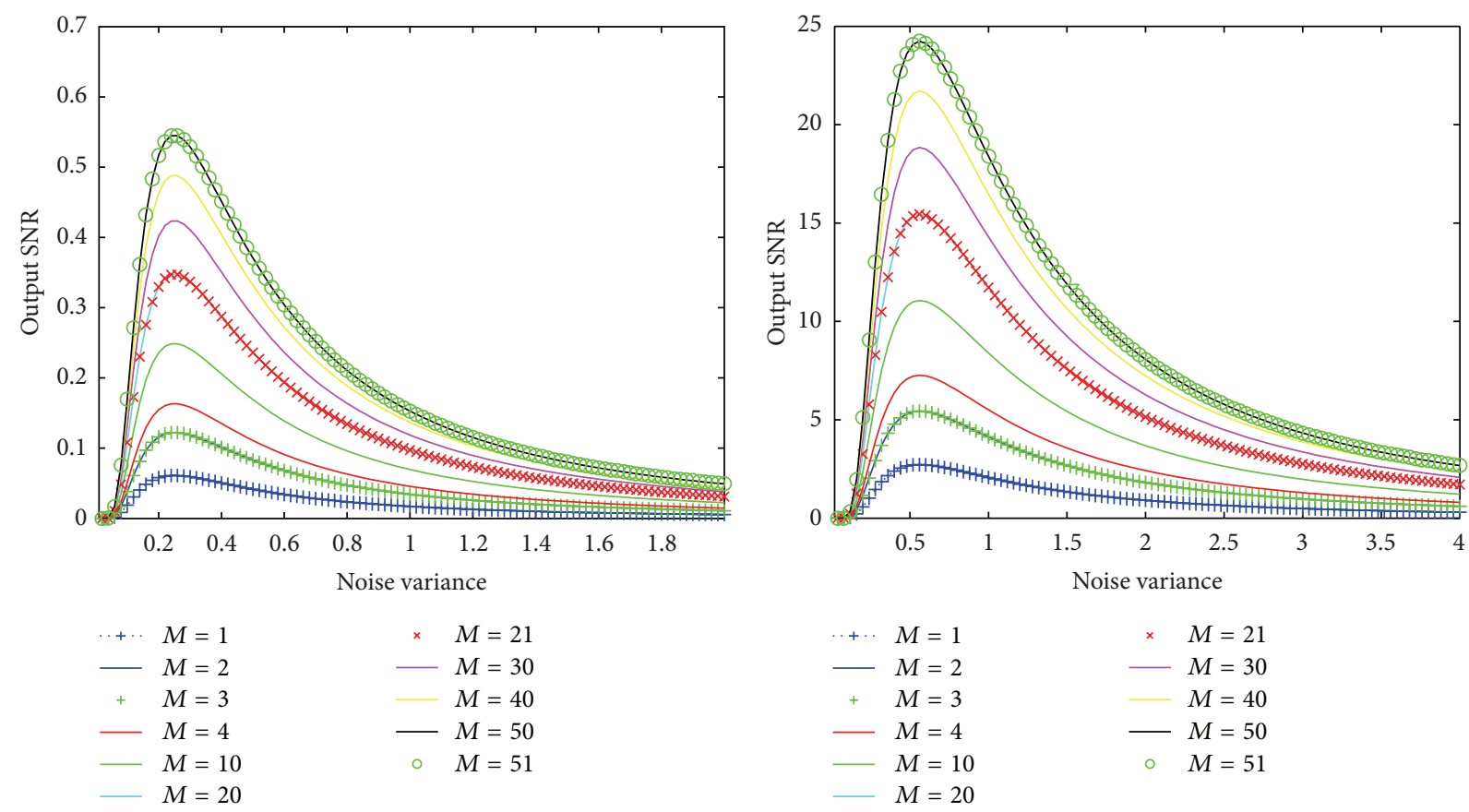

(a)

(b)

FIGURE 3: SNR curve changes as noise power. (a) $a=1, b=1, A=0.1$, and $f=100$. (b) $a=1.5, b=1, A=1$, and $f=10$.

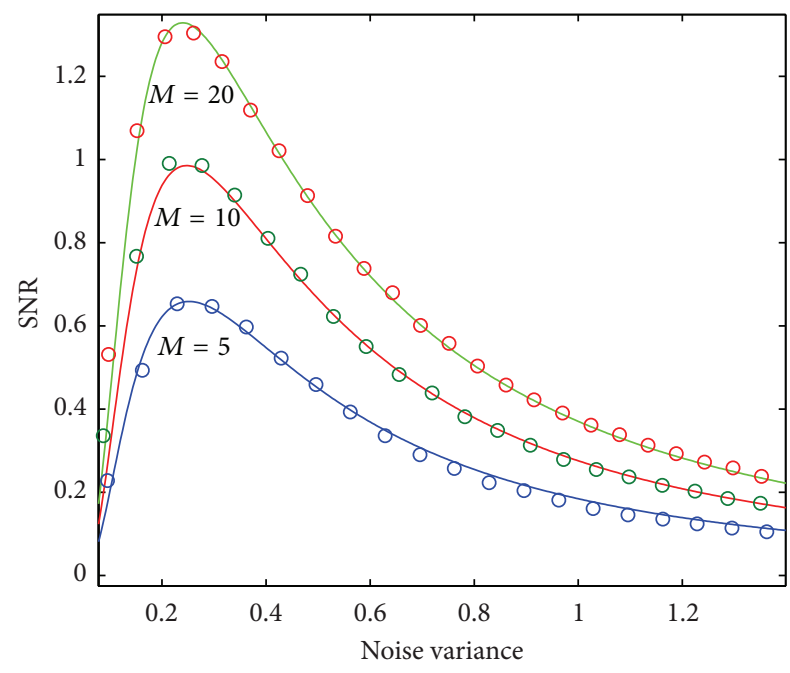

FIGURE 4: Output SNR with $a=1, b=1, A=0.02$, and $f=0.01$. The solid lines are from the theory of (8). The sets of discrete points (०) are from Monte Carlo simulations.

SNR between $M$ and $M+1$ array is very approximate leaving very small difference. This is because, for an even $M$, we have $(G(M+1)-F(M)) /(F(M+2)-G(M+1)) \approx 0$. Then $\operatorname{SNR}(M+1) / \operatorname{SNR}(M) \approx 1$. And the increment grows smaller even if $M$ is only odd or even with increasing $M$.

The results of Figure 3 reveal that the characteristic behaviors that identify the array bistable SR are precisely exhibited by the evolutions of the SNR. However for equal $M$, SNR displays different evolutions in the two figures in Figure 3. This is caused by the other parameters of the system. We will show the influence of these parameters in the following examples.

We also offered a validation by a Monte Carlo simulation of the proposed system in Figure 4 by setting $a=1, b=1$, $A=0.02$, and $f=0.01$. The results coincided with (8).

4.2. SR Effect and the Influence of Signal Amplitude. We consider, in Figure 5, the transmission by the array of a sinusoidal wave $s(t)=A_{0} \cos (2 \pi t / T s)$ buried in noise based on the theory of (8). The values of the amplitude $A_{0}$ determine how the input $s(t)$ is seen by the array. We choose a parallel array with $a=1, b=1, M=38$, and $f=100$. Figure 5 shows various evolutions of the SNR at the output of the array, for different values of the constant $A_{0}$. For the value of $A_{0}$ tested in Figure 5, the performance for the periodic input $s(t)$ is always SR. With increasing $A_{0}$, as the level of noise variance is increased, the output SNR experiences nonmonotonic evolutions. In this experiment, we also set $A_{0}$ to be very large numbers and very small numbers under $A_{0}>0$. The outputs of the system all perform the SR effect. And as $A_{0}$ grows, the effect gets more enhanced. We relate these results to the phenomenon of the proposed parallel array SR, by which nonlinear transmission or processing of signals with arbitrary amplitude can be improved by adding noises in arrays.

4.3. SR Effect and the Influence of Signal Frequency. In this example, we consider $A_{0}=0.1, a=1.5, b=1$, and $M=33$ array system based on the theory of (8). Let $f=\Omega / 2 \pi$. We choose different $f$ to see the influence of the signal frequency. 


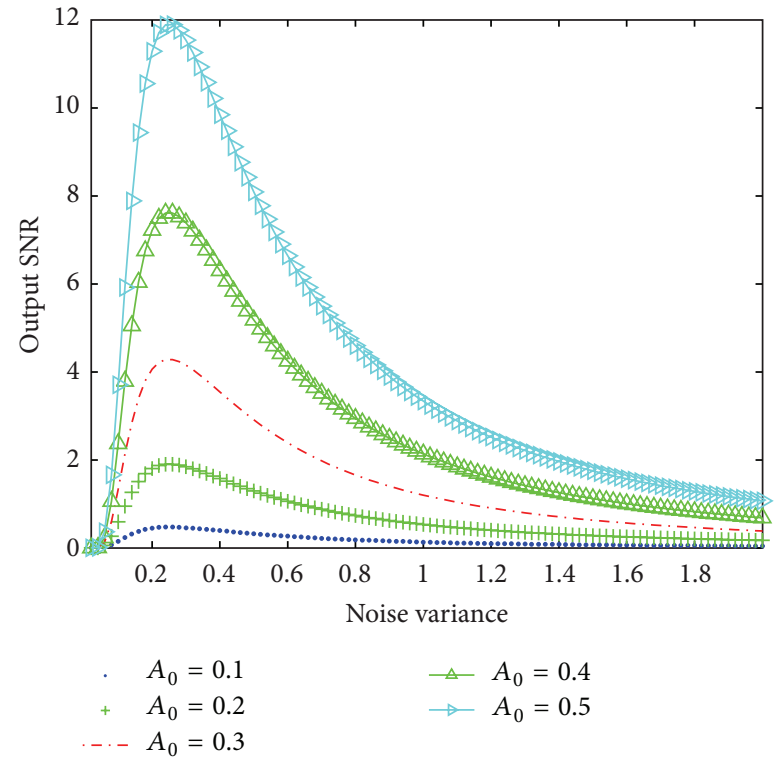

FIgURE 5: Output SNR as a function of noise variance with $a=1$, $b=1, M=38$, and $f=100$.

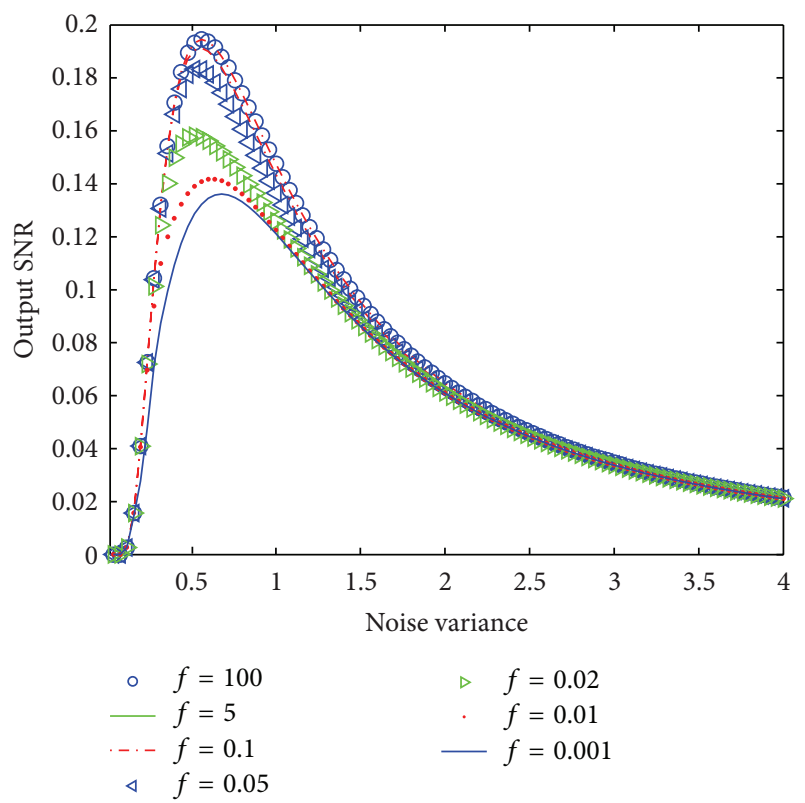

FIGURE 6: Output SNR as a function of noise variance with $A_{0}=0.1$, $a=1.5, b=1$, and $M=33$.

The output SNR versus noise variance of the two systems is given in Figure 6. We observe that the output SNR grows from near zero point to the maximum point and then goes down with different frequency tested in this example. The noise variances of maximum output SNR points in this case are slightly different. With the growing of frequency the SR effect becomes more enhanced. However, when $f$ is big enough, the growth of $f$ does not affect the output SNR. No matter how we increase the frequency, the system stays the same at the extremal SNR. This property also helps us to choose a suitable signal frequency under our hypothesis $\Delta t \ll 1 / f$. In our experiment when $f<0.2$ the system loses the SR effect. This phenomenon also shows that the system does not have the SR effect when the signal is DC signal, since we can take the periodic signal as DC signal, if the frequency of the signal is extremely low.

4.4. SR Effect and the Influence of System Parameters. Figure 7 shows various evolutions of the SNR at the output of the array, for different values of system parameters $a$ and $b$ based on the theory of (8). In Figure 7(a) to observe the influence of $a$, we set $A=0.1, b=1.5, f=10$, and $M=33$. As we can see from the figure, the smaller the parameter $a$ is, the stronger the SR effect is. When $a$ becomes big enough, the system loses the SR effect. In fact, in this condition the output SNR is nearly zero.

In Figure 7(b) to observe the influence of $b$, we set $A=$ $0.1, a=1.5, f=10$, and $M=33$, the same as Figure 7(a) except parameters $a$ and $b$. The result of the output SNR versus noise variance indicates that the bigger the parameter $b$, the stronger the SR effect. This is the opposite to the influence of $a$, because $b$ has a positive effect on reflectionsymmetric quartic potential, while $a$ has a negative one. And comparing the two figures, the influence of $a$ outweighs that of $b$, and this is obvious due to Kramers rate.

4.5. Input-Output SNR Gain. We still adopt the definition for input and output SNR in (5). Then input SNR for the sinusoidal signal and a zero-mean, Gaussian white noise $\mathrm{SNR}_{\mathrm{in}}=\pi A^{2} / D$. The input-output SNR gain is defined below. Consider

$$
G_{\mathrm{SNR}}=\frac{\mathrm{SNR}_{\mathrm{out}}}{\mathrm{SNR}_{\mathrm{in}}} .
$$

In this experiment, we let $A_{0}=0.1, a=1, b=1$, and $f=1$. In Figure 8 , the $G_{\text {SNR }}$ grows first and then decreases with increased noise variance. And the result in Figure 8 shows the array system outweighs the signal bistable system on SR effect and the $G_{\mathrm{SNR}}$ can exceed unity for $M>3$ in this experiment. It means that the array can improve the signal-to-noise ratio (SNR) by noise incoherently. The improvement is measured by the array gain. For $M=50$, the maximum of $G_{\mathrm{SNR}}$ is 2.95. Thus, this SR array with independent sensors provides a preferable strategy for processing periodic signals to the array without independent sensors which exceeds unity much less [19].

\section{Conclusion}

In this work, we study the design of structure of bistable system aimed at enhancing the SR effect to improve the performance, driven by sinusoidal signal and Gaussian white noise. We first proposed a parallel array bistable system with $M$ independent components and averaged output. We further deduced the output signal-to-noise ratio (SNR) for this parallel array system to analyse the performance of this SR system. Our examples not only show the proposed system reserves the SR property, but also give an analysis of different 

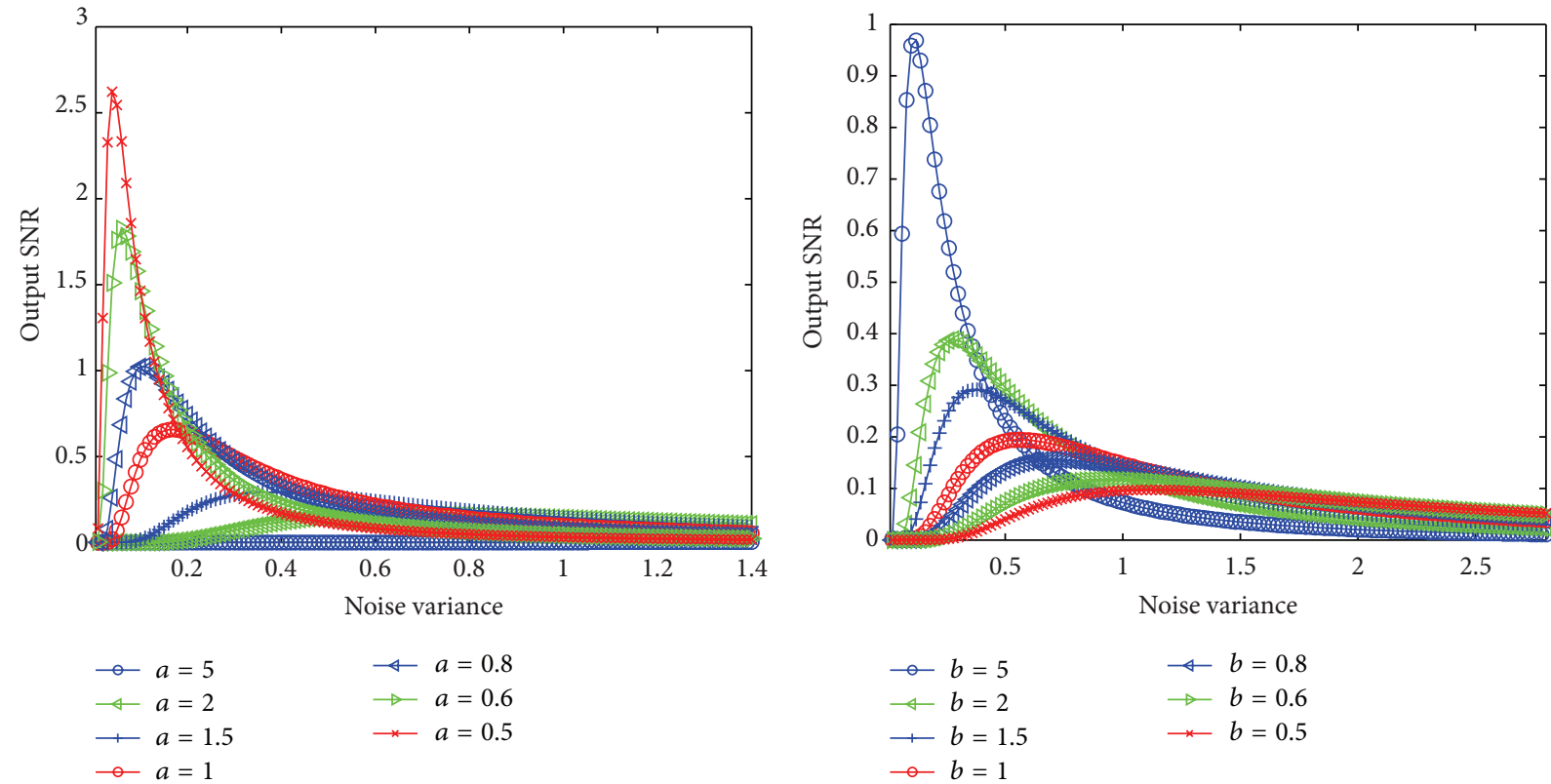

(a)

(b)

Figure 7: Output SNR as a function of noise variance. (a) $A=0.1, b=1.5, f=10$, and $M=33$. (b) $A=0.1, a=1.5, f=10$, and $M=33$.

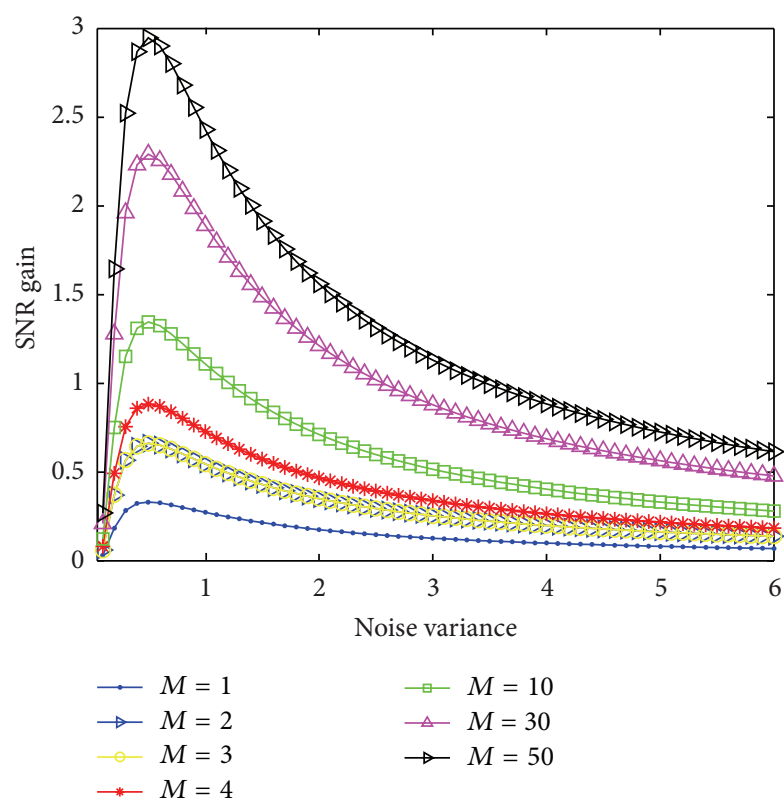

FIGURE 8: Output SNR gain as a function of noise variance, with $A_{0}=0.1, a=1, b=1$, and $f=1$.

parameter influences on the performance of the proposed parallel array, indicating a promising application in array signal processing.

\section{Appendices}

\section{A. Proofs of Lemmas 2-5 and Theorem 1}

Proof of Lemma 2. From the structure of the parallel array bistable system, the output of the system is

$$
z(t)=\frac{\sum_{i=1}^{M} x_{i}(t)}{M} .
$$

For the $i$ th bistable component, the pdf of $x_{i}(t)$ is

$$
p_{x}\left(x_{i}, t \mid x_{0}, t_{0}\right)=n_{+}(t) \delta\left(x_{i}-x_{m}\right)+n_{-}(t) \delta\left(x_{i}+x_{m}\right) .
$$

Since $x_{i}(t)$ is independent in statistics for $i=1,2, \ldots, M$, the pdf of the sum of the outputs of $M$ components is the convolution of each component. Then due to property of delta function, we simplify the result of the convolution and obtain the following pdf of $z(t)$. Consider

$$
\begin{aligned}
p_{z}\left(z, t \mid z_{0}, t_{0}\right) & \\
= & \sum_{m=0}^{M}\left(\begin{array}{l}
M \\
m
\end{array}\right) n_{+}^{m}(t) n_{-}^{M-m}(t) \delta\left(z-\frac{2 m-M}{M} x_{m}\right) .
\end{aligned}
$$

Proof of Lemma 3. Based on Lemma 2 and the general definition of autocorrelation function, we can deduce the autocorrelation function of $z(t)$,

$$
\begin{aligned}
& R_{z}(t+\tau, t) \\
& =\iint_{-\infty}^{+\infty} z_{1} z_{2} p_{z}\left(z_{1}, t+\tau \mid z_{2}, t\right) \\
& \quad \times p_{z}\left(z_{2}, t \mid z_{0}, t_{0}\right) d z_{1} d z_{2} \\
& =\iint_{-\infty}^{+\infty} z_{1} z_{2} \times \sum_{m=0}^{M}\left(\begin{array}{c}
M \\
m
\end{array}\right) n_{+}^{m}\left(t+\tau \mid z_{2}, t\right)
\end{aligned}
$$




$$
\begin{aligned}
& \times n_{-}^{M-m}\left(t+\tau \mid z_{2}, t\right) \\
& \times \delta\left(z_{1}-\frac{2 m-M}{M} x_{m}\right) \\
& \times \sum_{k=0}^{M}\left(\begin{array}{c}
M \\
k
\end{array}\right) n_{+}^{k}\left(t \mid z_{0}, t_{0}\right) \\
& \times n_{-}^{M-k}\left(t \mid z_{0}, t_{0}\right) \\
& \times \delta\left(z_{2}-\frac{2 k-M}{M} x_{m}\right) d z_{1} d z_{2} \\
& =x_{m}^{2} \sum_{m=0}^{M} \frac{2 m-M}{M}\left(\begin{array}{l}
M \\
m
\end{array}\right) \\
& \times n_{+}^{m}\left(t+\tau \mid \frac{2 k-M}{M} x_{m}, t\right) \\
& \times n_{-}^{M-m}\left(t+\tau \mid \frac{2 k-M}{M} x_{m}, t\right) \\
& \times \sum_{k=0}^{M} \frac{2 k-M}{M}\left(\begin{array}{c}
M \\
k
\end{array}\right) n_{+}^{k}\left(t \mid z_{0}, t_{0}\right) \\
& \times n_{-}^{M-k}\left(t \mid z_{0}, t_{0}\right) \text {. }
\end{aligned}
$$

The last step follows from the property of delta function.

Since

$$
\sum_{i=0}^{N}\left(\begin{array}{c}
N \\
i
\end{array}\right) \frac{2 i-N}{N} x^{i} y^{N-i}=(x+y)^{N-1}(x-y)
$$

and making use of the normalization condition $n_{+}(t)+n_{-}(t)=$ 1, (A.4) becomes

$$
\begin{aligned}
R_{z}(t+\tau, t) & \\
=x_{m}^{2}\left[n_{+}\left(t+\tau \mid \frac{2 k-M}{M} x_{m}, t\right)\right. & \left.\quad n_{-}\left(t+\tau \mid \frac{2 k-M}{M} x_{m}, t\right)\right] \\
& \times \sum_{k=0}^{M} \frac{2 k-M}{M}\left(\begin{array}{c}
M \\
k
\end{array}\right) n_{+}^{k}\left(t \mid z_{0}, t_{0}\right) n_{-}^{M-k}\left(t \mid z_{0}, t_{0}\right) \\
= & x_{m}^{2}\left[2 n_{+}\left(t+\tau \mid \frac{2 k-M}{M} x_{m}, t\right)-1\right] \\
& \times \sum_{k=0}^{M} \frac{2 k-M}{M}\left(\begin{array}{c}
M \\
k
\end{array}\right) n_{+}^{k}\left(t \mid z_{0}, t_{0}\right) n_{-}^{M-k}\left(t \mid z_{0}, t_{0}\right) .
\end{aligned}
$$

If $M$ is even, the range $(0, M)$ of $k$ can be divided into $(0, M / 2-1)$ and $(M / 2, M)$. If $k=M / 2$, it is obvious that $R_{z}(t+\tau, t)=0$. Then

$$
\begin{aligned}
& R_{z}(t+\tau, t) \\
& =x_{m}^{2}\left\{\left[2 n_{+}(t+\tau \mid-, t)-1\right]\right. \\
& \times \sum_{k=0}^{M / 2-1} \frac{2 k-M}{M}\left(\begin{array}{c}
M \\
k
\end{array}\right) n_{+}^{k}\left(t \mid z_{0}, t_{0}\right) \\
& \times n_{-}^{M-k}\left(t \mid z_{0}, t_{0}\right) \\
& +\left[2 n_{+}(t+\tau \mid+, t)-1\right] \\
& \times \sum_{k=M / 2+1}^{M} \frac{2 k-M}{M}\left(\begin{array}{c}
M \\
k
\end{array}\right) n_{+}^{k}\left(t \mid z_{0}, t_{0}\right) \\
& \left.\times n_{-}^{M-k}\left(t \mid z_{0}, t_{0}\right)\right\} .
\end{aligned}
$$

From [21], we have

$$
\begin{aligned}
n_{+} & (t+\tau \mid-, t) \\
= & \frac{1}{2}\left\{\exp \left(-2 r_{k}|\tau|\right)[-1-\kappa(t)]+1+\kappa(t+\tau)\right\}, \\
n_{+} & (t+\tau \mid+, t) \\
\quad & \frac{1}{2}\left\{\exp \left(-2 r_{k}|\tau|\right)[1-\kappa(t)]+1+\kappa(t+\tau)\right\},
\end{aligned}
$$

where

$$
\begin{gathered}
\kappa(t)=B \cos (\Omega t-\varphi), \\
B=\frac{2 r_{k}\left(A_{0} x_{m} / D\right)}{\sqrt{\left(4 r_{k}^{2}+\Omega^{2}\right)}} .
\end{gathered}
$$

$n_{+}(t+\tau \mid-, t)$ and $n_{+}(t+\tau \mid+, t)$ are $\alpha<0$ and $\alpha>0$ in $n_{+}(t+\tau \mid \alpha, t)$, respectively [21].

It greatly simplifies in the stationary limit $t_{0} \rightarrow-\infty$,

$$
\begin{aligned}
\lim _{t_{0} \rightarrow-\infty} R_{z}(t+\tau, t) \\
=x_{m}^{2}\left\{\left\{\exp \left(-2 r_{k}|\tau|\right)[-1-\kappa(t)]+\kappa(t+\tau)\right\}\right. \\
\quad \times \sum_{k=0}^{M / 2-1} \frac{2 k-M}{M}\left(\begin{array}{c}
M \\
k
\end{array}\right) n_{+}^{k}\left(t \mid z_{0}, t_{0}\right) \\
\quad \times n_{-}^{M-k}\left(t \mid z_{0}, t_{0}\right) \\
+\left\{\exp \left(-2 r_{k}|\tau|\right)[1-\kappa(t)]+\kappa(t+\tau)\right\} \\
\quad \times \sum_{k=M / 2+1}^{M} \frac{2 k-M}{M}\left(\begin{array}{c}
M \\
k
\end{array}\right) n_{+}^{k}\left(t \mid z_{0}, t_{0}\right)
\end{aligned}
$$




$$
\begin{aligned}
& \left.\times n_{-}^{M-k}\left(t \mid z_{0}, t_{0}\right)\right\} \\
& \left.\times\left\{\frac{1}{2}[1-\kappa(t)]\right\}^{M-k}\right\} \\
& =x_{m}^{2}\left\{\left[-\exp \left(-2 r_{k}|\tau|\right) \kappa^{2}(t)+\kappa(t+\tau)\right] \kappa(t)\right. \\
& -\exp \left(-2 r_{k}|\tau|\right) \\
& \times \sum_{k=0}^{M / 2-1} \frac{2 k-M}{M}\left(\begin{array}{c}
M \\
k
\end{array}\right) n_{+}^{k}\left(t \mid z_{0}, t_{0}\right) \\
& \times n_{-}^{M-k}\left(t \mid z_{0}, t_{0}\right) \\
& +\exp \left(-2 r_{k}|\tau|\right) \\
& \times \sum_{k=M / 2+1}^{M} \frac{2 k-M}{M}\left(\begin{array}{c}
M \\
k
\end{array}\right) n_{+}^{k}\left(t \mid z_{0}, t_{0}\right) \\
& \left.\times n_{-}^{M-k}\left(t \mid z_{0}, t_{0}\right)\right\} \text {. } \\
& =x_{m}^{2}\left\{\left[-\exp \left(-2 r_{k}|\tau|\right) \kappa^{2}(t)+\kappa(t+\tau)\right] \kappa(t)\right. \\
& -\left(\frac{1}{2}\right)^{M} \exp \left(-2 r_{k}|\tau|\right) \\
& \times\left\{\sum_{k=0}^{M / 2-1} \frac{2 k-M}{M}\left(\begin{array}{c}
M \\
k
\end{array}\right)[1+\kappa(t)]^{k}\right. \\
& \times[1-\kappa(t)]^{M-k} \\
& -\sum_{k=M / 2+1}^{M} \frac{2 k-M}{M}\left(\begin{array}{c}
M \\
k
\end{array}\right) \\
& \times[1+\kappa(t)]^{k} \\
& \left.\left.\times[1-\kappa(t)]^{M-k}\right\}\right\} .
\end{aligned}
$$

Take

$$
\begin{aligned}
& \lim _{t_{0} \rightarrow-\infty} n_{+}\left(t \mid z_{0}, t_{0}\right)=\frac{1}{2}[1+\kappa(t)], \\
& \lim _{t_{0} \rightarrow-\infty} n_{-}\left(t \mid z_{0}, t_{0}\right)=\frac{1}{2}[1-\kappa(t)]
\end{aligned}
$$

into (A.10) to obtain

$$
\begin{aligned}
\lim _{t_{0} \rightarrow-\infty} R_{z}( & t+\tau, t) \\
=x_{m}^{2}\{[ & \left.-\exp \left(-2 r_{k}|\tau|\right) \kappa^{2}(t)+\kappa(t+\tau)\right] \kappa(t) \\
& -\exp \left(-2 r_{k}|\tau|\right) \\
& \times \sum_{k=0}^{M / 2-1} \frac{2 k-M}{M}\left(\begin{array}{c}
M \\
k
\end{array}\right)\left\{\frac{1}{2}[1+\kappa(t)]\right\}^{k} \\
& \times\left\{\frac{1}{2}[1-\kappa(t)]\right\}^{M-k} \\
& +\exp \left(-2 r_{k}|\tau|\right) \\
& \times \sum_{k=M / 2+1}^{M} \frac{2 k-M}{M}\left(\begin{array}{c}
M \\
k
\end{array}\right) \\
& \times\left\{\frac{1}{2}[1+\kappa(t)]\right\}^{k}
\end{aligned}
$$

The last part can be reexpressed as

$$
\begin{aligned}
& \sum_{k=0}^{M / 2-1} \frac{2 k-M}{M}\left(\begin{array}{c}
M \\
k
\end{array}\right)[1+\kappa(t)]^{k}[1-\kappa(t)]^{M-k} \\
& -\sum_{k=0}^{M / 2-1} \frac{M-2 k}{M}\left(\begin{array}{c}
M \\
M-k
\end{array}\right)[1+\kappa(t)]^{M-k}[1-\kappa(t)]^{k} \\
& =\sum_{k=0}^{M / 2-1} \frac{2 k-M}{M}\left(\begin{array}{c}
M \\
k
\end{array}\right) \\
& \times \sum_{i=0}^{k} \sum_{j=0}^{M-k}\left(\begin{array}{l}
k \\
i
\end{array}\right)\left(\begin{array}{c}
M-k \\
j
\end{array}\right) \kappa(t)^{M-i-j} \\
& \times\left[(-1)^{M-k-j}+(-1)^{k-i}\right] \\
& =\sum_{k=0}^{M / 2-1} \frac{2 k-M}{M}\left(\begin{array}{c}
M \\
k
\end{array}\right) \\
& \times \sum_{i=0}^{k} \sum_{j=0}^{M-k}\left(\begin{array}{c}
k \\
i
\end{array}\right)\left(\begin{array}{c}
M-k \\
j
\end{array}\right) B^{M-i-j} \\
& \times \cos (\Omega t-\varphi)^{M-i-j} \\
& \times\left[(-1)^{M-k-j}+(-1)^{k-i}\right] \text {. }
\end{aligned}
$$


Then we have

$$
\begin{aligned}
& R_{z}(t+\tau, t) \\
&=x_{m}^{2}\left\{\left[-\exp \left(-2 r_{k}|\tau|\right) \kappa^{2}(t)+\kappa(t+\tau)\right] \kappa(t)\right. \\
&-\left(\frac{1}{2}\right)^{M} \exp \left(-2 r_{k}|\tau|\right) \\
& \times \sum_{k=0}^{M / 2-1} \frac{2 k-M}{M}\left(\begin{array}{c}
M \\
k
\end{array}\right) \\
& \times \sum_{i=0}^{k} \sum_{j=0}^{M-k}\left(\begin{array}{c}
k \\
i
\end{array}\right)\left(\begin{array}{c}
M-k \\
j
\end{array}\right) B^{M-i-j} \\
& \times \cos (\Omega t-\varphi)^{M-i-j} \\
&\left.\times\left[(-1)^{M-k-j}+(-1)^{k-i}\right]\right\} .
\end{aligned}
$$

Proof of Lemma 4. If $M$ is odd, we can prove Lemma 4 in a similar manner as Lemma 3. After some mathematical manipulations, we obtain the following:

$$
\begin{aligned}
R_{z}(t+\tau, t) & \\
=x_{m}^{2}\{[ & \left.-\exp \left(-2 r_{k}|\tau|\right) \kappa^{2}(t)+\kappa(t+\tau)\right] \kappa(t) \\
& -\left(\frac{1}{2}\right)^{M} \exp \left(-2 r_{k}|\tau|\right) \\
& \times \sum_{k=0}^{M-1 / 2} \frac{2 k-M}{M}\left(\begin{array}{c}
M \\
k
\end{array}\right) \\
& \times \sum_{i=0}^{k} \sum_{j=0}^{M-k}\left(\begin{array}{c}
k \\
i
\end{array}\right)\left(\begin{array}{c}
M-k \\
j
\end{array}\right) B^{M-i-j} \\
& \times \cos (\Omega t-\varphi)^{M-i-j} \\
& \left.\times\left[(-1)^{M-i-j}+(-1)^{k-i}\right]\right\}
\end{aligned}
$$

Proof of Lemma 5. It is obvious that the autocorrelation function depends on both times $t+\tau$ and $t$. However, in real experiments $t$ represents the time set for the trigger in the data acquisition procedure. Typically, the averages implied by the definition of the autocorrelation function are taken over many sampling records of the signal $x(t)$, triggered at a large number of times $t$ within one period of the forcing $T_{\Omega}$. Hence, the corresponding phases of the input signal, $\theta=\Omega t+\psi$, are uniformly distributed between 0 and $2 \pi$. This corresponds to averaging autocorrelation function as with respect to $t$ uniformly over an entire forcing period, whence if $M$ is even,

$$
\begin{aligned}
& R_{z}(\tau) \\
& =\frac{1}{T_{\Omega}} \int_{0}^{T_{\Omega}} R_{z}(t+\tau, t) d t \\
& =\frac{1}{T_{\Omega}} \int_{0}^{T_{\Omega}} x_{m}^{2} \\
& \times\left\{\left[-\exp \left(-2 r_{k}|\tau|\right) \kappa^{2}(t)+\kappa(t+\tau)\right] \kappa(t)\right. \\
& -\left(\frac{1}{2}\right)^{M} \exp \left(-2 r_{k}|\tau|\right) \\
& \times \sum_{k=0}^{M / 2-1} \frac{2 k-M}{M}\left(\begin{array}{c}
M \\
k
\end{array}\right) \\
& \times \sum_{i=0}^{k} \sum_{j=0}^{M-k}\left(\begin{array}{c}
k \\
i
\end{array}\right)\left(\begin{array}{c}
M-k \\
j
\end{array}\right) B^{M-i-j} \\
& \times \cos (\Omega t-\varphi)^{M-i-j} \\
& \left.\times\left[(-1)^{M-k-j}+(-1)^{k-i}\right]\right\} d t \\
& =x_{m}^{2}\left\{-\frac{1}{2} \exp \left(-2 r_{k}|\tau|\right) B^{2}\right. \\
& +\frac{1}{2} B^{2} \cos (\Omega \tau)-\left(\frac{1}{2}\right)^{M} \exp \left(-2 r_{k}|\tau|\right) \\
& \times \sum_{k=0}^{M / 2-1} \frac{2 k-M}{M} \\
& \times\left(\begin{array}{c}
M \\
k
\end{array}\right) \sum_{i=0}^{k} \sum_{j=0}^{M-k}\left(\begin{array}{c}
k \\
i
\end{array}\right)\left(\begin{array}{c}
M-k \\
j
\end{array}\right) \\
& \times B^{M-i-j} f(M-i-j) \\
& \left.\times\left[(-1)^{M-k-j}+(-1)^{k-i}\right]\right\}
\end{aligned}
$$

in which

$$
\begin{aligned}
& f(x)=\frac{\Gamma((x+1) / 2)}{\sqrt{\pi} \Gamma(x / 2+1)}, \\
& \Gamma(x)=\int_{0}^{\infty} t^{x-1} e^{-t} d t .
\end{aligned}
$$


Then

$$
R_{z}(\tau)
$$

$$
\begin{aligned}
=x_{m}^{2}\{ & -\exp \left(-2 r_{k}|\tau|\right) \frac{1}{2} B^{2} \\
& \left.+\frac{1}{2} B^{2} \cos (\Omega \tau)-\exp \left(-2 r_{k}|\tau|\right) F(M)\right\},
\end{aligned}
$$

where

$$
\begin{aligned}
& F(M) \\
& =\left(\frac{1}{2}\right)^{M} \sum_{k=0}^{M / 2-1} \frac{2 k-M}{M}\left(\begin{array}{c}
M \\
k
\end{array}\right) \\
& \quad \times \sum_{i=0}^{k} \sum_{j=0}^{M-k}\left(\begin{array}{c}
k \\
i
\end{array}\right)\left(\begin{array}{c}
M-k \\
j
\end{array}\right) \\
& \quad \times B^{M-i-j} f(M-i-j) \\
& \quad \times\left[(-1)^{M-k-j}+(-1)^{k-i}\right] .
\end{aligned}
$$

Using Fourier transform of (A.18), we obtain the power spectrum density under the condition that $M$ is even. Consider

$$
\begin{aligned}
S(\omega)= & \frac{4 r_{k} x_{m}^{2}}{4 r_{k}^{2}+\omega^{2}}\left(-\frac{1}{2} B^{2}\right) \\
& +\frac{\pi}{2} x_{m}^{2} B^{2}[\delta(\omega-\Omega)+\delta(\omega+\Omega)] \\
& -\frac{4 r_{k} x_{m}^{2}}{4 r_{k}^{2}+\omega^{2}} F(M) \\
= & -\left[\frac{1}{2}\left(\frac{A_{0} x_{m}}{D}\right)^{2} \frac{4 r_{k}^{2}}{4 r_{k}^{2}+\Omega^{2}}+F(M)\right] \frac{4 r_{k} x_{m}^{2}}{4 r_{k}^{2}+\omega^{2}} \\
& +\frac{\pi}{2}\left(\frac{A_{0} x_{m}}{D}\right)^{2} \frac{4 r_{k}^{2} x_{m}^{2}}{4 r_{k}^{2}+\Omega^{2}}[\delta(\omega-\Omega)+\delta(\omega+\Omega)] .
\end{aligned}
$$

If $M$ is odd, the method is similar. Consider

$$
\begin{aligned}
& R_{z}(\tau) \\
& =\frac{1}{T_{\Omega}} \int_{0}^{T_{\Omega}} R_{z}(t+\tau, t) d t \\
& =\frac{1}{T_{\Omega}} \\
& \quad \times \int_{0}^{T_{\Omega}} x_{m}^{2} \\
& \times\left\{\left[-\exp \left(-2 r_{k}|\tau|\right) \kappa^{2}(t)\right.\right. \\
& \quad+\kappa(t+\tau)] \kappa(t)
\end{aligned}
$$$$
-\left(\frac{1}{2}\right)^{M} \exp \left(-2 r_{k}|\tau|\right)
$$$$
\times \sum_{k=0}^{(M-1) / 2} \frac{2 k-M}{M}\left(\begin{array}{c}
M \\
k
\end{array}\right)
$$$$
\times \sum_{i=0}^{k} \sum_{j=0}^{M-k}\left(\begin{array}{c}
k \\
i
\end{array}\right)\left(\begin{array}{c}
M-k \\
j
\end{array}\right)
$$$$
\times B^{M-i-j} \cos (\Omega t-\varphi)^{M-i-j}
$$$$
\left.\times\left[(-1)^{M-i-j}+(-1)^{k-i}\right]\right\} d t
$$$$
=x_{m}^{2}\left\{-\frac{1}{2} \exp \left(-2 r_{k}|\tau|\right) \frac{1}{2} B^{2}\right.
$$$$
+\frac{1}{2} B^{2} \cos (\Omega \tau)-\left(\frac{1}{2}\right)^{M} \exp \left(-2 r_{k}|\tau|\right)
$$$$
\times \sum_{k=0}^{(M-1) / 2} \frac{2 k-M}{M}
$$$$
\times\left(\begin{array}{c}
M \\
k
\end{array}\right) \sum_{i=0}^{k} \sum_{j=0}^{M-\mathrm{k}}\left(\begin{array}{c}
k \\
i
\end{array}\right)\left(\begin{array}{c}
M-k \\
j
\end{array}\right)
$$$$
\times B^{M-i-j} f(M-i-j)
$$$$
\left.\times\left[(-1)^{M-i-j}+(-1)^{k-i}\right]\right\},
$$

in which $f(x)$ and $\Gamma(x)$ have the same definition in (A.17). Then

$$
\begin{aligned}
R_{z}(\tau)=x_{m}^{2}\{ & -\frac{1}{2} \exp \left(-2 r_{k}|\tau|\right) B^{2} \\
& \left.+\frac{1}{2} B^{2} \cos (\Omega \tau)-\exp \left(-2 r_{k}|\tau|\right) G(M)\right\},
\end{aligned}
$$

in which

$$
\begin{aligned}
& G(M) \\
& =\left(\frac{1}{2}\right)^{M} \sum_{k=0}^{M-1) / 2} \frac{2 k-M}{M}\left(\begin{array}{c}
M \\
k
\end{array}\right) \\
& \quad \times \sum_{i=0}^{k M-k} \sum_{j=0}^{M}\left(\begin{array}{c}
k \\
i
\end{array}\right)\left(\begin{array}{c}
M-k \\
j
\end{array}\right) \\
& \quad \times B^{M-i-j} f(M-i-j) \\
& \quad \times\left[(-1)^{M-i-j}+(-1)^{k-i}\right] .
\end{aligned}
$$


The Fourier transform of power spectrum density is

$$
\begin{aligned}
S(\omega)= & \frac{4 r_{k} x_{m}^{2}}{4 r_{k}^{2}+\omega^{2}}\left(-\frac{1}{2} B^{2}\right) \\
& +\frac{\pi}{2} x_{m}^{2} B^{2}[\delta(\omega-\Omega)+\delta(\omega+\Omega)] \\
& -\frac{4 r_{k} x_{m}^{2}}{4 r_{k}^{2}+\omega^{2}} G(M) \\
= & -\left[\frac{1}{2}\left(\frac{A_{0} x_{m}}{D}\right)^{2} \frac{4 r_{k}^{2}}{4 r_{k}^{2}+\Omega^{2}}+G(M)\right] \frac{4 r_{k} x_{m}^{2}}{4 r_{k}^{2}+\omega^{2}} \\
& +\frac{\pi}{2}\left(\frac{A_{0} x_{m}}{D}\right)^{2} \frac{4 r_{k}^{2} x_{m}^{2}}{4 r_{k}^{2}+\Omega^{2}} \\
& \times[\delta(\omega-\Omega)+\delta(\omega+\Omega)] .
\end{aligned}
$$

In conclusion, the power spectrum density of the output of the system is

$S(\omega)$

$$
\begin{aligned}
= & -\left[\frac{1}{2}\left(\frac{A_{0} x_{m}}{D}\right)^{2} \frac{4 r_{k}^{2}}{4 r_{k}^{2}+\Omega^{2}}+H(M)\right] \frac{4 r_{k} x_{m}^{2}}{4 r_{k}^{2}+\omega^{2}} \\
& +\frac{\pi}{2}\left(\frac{A_{0} x_{m}}{D}\right)^{2} \frac{4 r_{k}^{2} x_{m}^{2}}{4 r_{k}^{2}+\omega^{2}} \\
& \times[\delta(\omega-\Omega)+\delta(\omega+\Omega)],
\end{aligned}
$$

in which

$$
H(M)= \begin{cases}F(M), & M \text { is even number } \\ G(M), & M \text { is odd number. }\end{cases}
$$

Proof of Theorem 1. In (A.25), we can easily separate an exponentially decaying branch due to randomness and a periodically oscillating tail driven by the periodic input signal. And as a matter of fact, power spectrum density of noise $S_{N}(\omega)$ is the product of the Lorentzian curve obtained with no input signal $A_{0}=0$ and a factor that depends on the forcing amplitude $A_{0}$, but it is smaller than unity. Then the first part of power spectrum density is caused by noise. Consider

$$
S_{N}(\omega)=-\left[\frac{1}{2}\left(\frac{A_{0} x_{m}}{D}\right)^{2} \frac{4 r_{k}^{2}}{4 r_{k}^{2}+\Omega^{2}}+H(M)\right] \frac{4 r_{k} x_{m}^{2}}{4 r_{k}^{2}+\omega^{2}},
$$

based on the definition of output SNR in (5).

Then for the parallel bistable system with $M$ components, the output SNR following the definition in (5) is

$$
\mathrm{SNR}=\frac{\pi\left(A_{0} x_{m} / D\right)^{2} r_{k}}{-(1 / 2)\left(A_{0} x_{m} / D\right)^{2}\left(4 r_{k}^{2} /\left(4 r_{k}^{2}+\Omega^{2}\right)\right)-H(M)} \text {. }
$$

\section{B. Proof of Theorem 6}

Proof of Theorem 6. The proof of Theorem 6 is similar to that of Theorem 1; an outline is provided as follows. The output in this system is

$$
z(t)=\frac{x_{1}(t)+x_{2}(t)}{2}
$$

The pdfs of $x_{i}$ for $i=1,2$ are

$$
p_{x}\left(x_{i}, t \mid x_{0}, t_{0}\right)=n_{+}(t) \delta\left(x_{i}-x_{m}\right)+n_{-}(t) \delta\left(x_{i}+x_{m}\right) .
$$

Then the pdf of $z(t)$ is

$$
p_{z}\left(z, t \mid x_{0}, t_{0}\right)=2\left[p_{x}\left(2 z, t \mid x_{0}, t_{0}\right) * p_{x}\left(2 z, t \mid x_{0}, t_{0}\right)\right] .
$$

Then

$$
\begin{aligned}
p_{z}\left(z, t \mid x_{0}, t_{0}\right)= & n_{+}(t)^{2} \delta\left(z-x_{m}\right) \\
& +2 n_{+}(t) n_{-}(t) \delta(z)+n_{-}(t)^{2} \delta\left(z+x_{m}\right) .
\end{aligned}
$$

According to the general definition of autocorrelation function, the autocorrelation function of $z(t)$ is

$$
\begin{aligned}
& R_{z}(t+\tau, t) \\
& =E[z(t+\tau) z(t)] \\
& =\iint_{-\infty}^{+\infty} z_{1} z_{2} p_{z}\left(z_{1}, t+\tau \mid z_{2}, t\right) \\
& \quad \times p_{z}\left(z_{2}, t \mid z_{0}, t_{0}\right) d z_{1} d z_{2} \\
& =\iint_{-\infty}^{+\infty} z_{1} z_{2} \quad \begin{array}{c}
\times\left[n_{+}\left(t+\tau \mid z_{2}, t\right)^{2} \delta\left(z_{1}-x_{m}\right)\right. \\
+2 n_{+}\left(t+\tau \mid z_{2}, t\right) n_{-}\left(t+\tau \mid z_{2}, t\right) \delta\left(z_{1}\right) \\
\left.+n_{-}\left(t+\tau \mid z_{2}, t\right)^{2} \delta\left(z_{1}+x_{m}\right)\right] \\
\times\left[n_{+}\left(t \mid z_{0}, t_{0}\right)^{2} \delta\left(z_{2}-x_{m}\right)\right. \\
+2 n_{+}\left(t \mid z_{0}, t_{0}\right) n_{-}\left(t \mid z_{0}, t_{0}\right) \delta\left(z_{2}\right) \\
\left.+n_{-}\left(t \mid z_{0}, t_{0}\right)^{2} \delta\left(z_{2}+x_{m}\right)\right] d z_{1} d z_{2} .
\end{array}
\end{aligned}
$$


Due to the property of delta function, we obtain

$$
\begin{aligned}
& R_{z}(t+\tau, t) \\
& =x_{m}^{2} n_{+}\left(t+\tau \mid x_{m}, t\right)^{2} n_{+}\left(t \mid z_{0}, t_{0}\right)^{2} \\
& \quad-x_{m}^{2} n_{+}\left(t+\tau \mid-x_{m}, t\right)^{2} n_{-}\left(t \mid z_{0}, t_{0}\right)^{2} \\
& \quad-x_{m}^{2} n_{-}\left(t+\tau \mid x_{m}, t\right)^{2} n_{+}\left(t \mid z_{0}, t_{0}\right)^{2} \\
& +x_{m}^{2} n_{-}\left(t+\tau \mid-x_{m}, t\right)^{2} n_{-}\left(t \mid z_{0}, t_{0}\right)^{2} \\
& =x_{m}^{2}\left\{n_{+}\left(t \mid z_{0}, t_{0}\right)^{2}\right. \\
& \quad \times\left[2 n_{+}\left(t+\tau \mid x_{m}, t\right)-2 n_{+}\left(t+\tau \mid-x_{m}, t\right)\right] \\
& \quad+\left[1-2 n_{+}\left(t+\tau \mid-x_{m}, t\right)\right] \\
& \left.\quad \times\left[1-2 n_{+}\left(t \mid z_{0}, t_{0}\right)\right]\right\} .
\end{aligned}
$$

Simplify it in the stationary limit $t_{0} \rightarrow-\infty$,

$$
\begin{aligned}
& \lim _{t_{0}} \rightarrow-\infty R_{z}(t+\tau, t) \\
& \quad=x_{m}^{2} \exp \left(-2 r_{k}|\tau|\right)\left[\frac{1-\kappa(t)^{2}}{2}\right]+x_{m}^{2} \kappa(t+\tau) \kappa(t) .
\end{aligned}
$$

And to obtain the average autocorrelation function

$$
\begin{aligned}
R_{z}(\tau)= & \left(\frac{1}{T_{\Omega}}\right) \int_{0}^{T_{\Omega}} R_{z}(t+\tau, t) d t \\
= & \frac{x_{m}^{2}}{2} \exp \left(-2 r_{k}|\tau|\right)\left[1-\frac{1}{2}\left(\frac{A_{0} x_{m}}{D}\right)^{2} \frac{4 r_{k}^{2}}{4 r_{k}^{2}+\Omega^{2}}\right] \\
& +\frac{x_{m}^{2}}{2}\left(\frac{A_{0} x_{m}}{D}\right)^{2} \frac{4 r_{k}^{2}}{4 r_{k}^{2}+\Omega^{2}} \cos (\Omega \tau) .
\end{aligned}
$$

The output power spectrum density is as follows:

$$
\begin{aligned}
S(\omega)= & \frac{1}{2}\left[1-\frac{1}{2}\left(\frac{A_{0} x_{m}}{D}\right)^{2} \frac{4 r_{k}^{2}}{4 r_{k}^{2}+\Omega^{2}}\right] \frac{4 r_{k} x_{m}^{2}}{4 r_{k}^{2}+\omega^{2}} \\
& +\frac{\pi}{2}\left(\frac{A_{0} x_{m}}{D}\right)^{2} \frac{4 r_{k}^{2} x_{m}^{2}}{4 r_{k}^{2}+\Omega^{2}}[\delta(\omega-\Omega)+\delta(\omega+\Omega)] .
\end{aligned}
$$

The first part is due to the noise, and then the power spectrum density of noise is

$$
S_{N}(\omega)=\frac{1}{2}\left[1-\frac{1}{2}\left(\frac{A_{0} x_{m}}{D}\right)^{2} \frac{4 r_{k}^{2}}{4 r_{k}^{2}+\Omega^{2}}\right] \frac{4 r_{k} x_{m}^{2}}{4 r_{k}^{2}+\omega^{2}}
$$

The system output SNR is

$$
\mathrm{SNR}=2 \pi\left(\frac{A_{0} x_{m}}{D}\right)^{2} r_{k}+O\left(A_{0}^{4}\right) .
$$

Omitting the high order items due to the weak signal, the SNR following the definition in (5) becomes

$$
\mathrm{SNR}=2 \pi\left(\frac{A_{0} x_{m}}{D}\right)^{2} r_{k}
$$

\section{Conflict of Interests}

The authors declare that there is no conflict of interests regarding the publication of this paper.

\section{Acknowledgments}

This work was supported by the National Natural Science Foundation of China, Grant no. 61102157, and the National Basic Research Program of China (973 Program), Grant no. 2013 CB329003.

\section{References}

[1] R. Benzi, A. Sutera, and A. Vulpiani, "The mechanism of stochastic resonance," Journal of Physics A, vol. 14, no. 11, pp. L453-L457, 1981.

[2] R. Benzi, G. Parisi, and A. Sutera, "Stochastic resonance in climatic change," Tellus, vol. 34, pp. 10-16, 1982.

[3] R. Benzi, G. Parisi, and A. Sutera, "A theory of s tochastic resonance in climatic change," SIAM Journal on Applied Mathematics, vol. 43, no. 3, pp. 565-578, 1983.

[4] A. A. Saha and G. V. Anand, "Design of detectors based on stochastic resonance," Signal Processing, vol. 83, no. 6, pp. 11931212, 2003.

[5] S. M. Saberali and H. Amindavar, "Analytical investigation of stochastic resonance phenomenon in linear detectors and comparison to maximum likelihood detector with application in co-channel interferer channels," IET Signal Processing, vol. 7, no. 2, pp. 128-133, 2013.

[6] D. He, "Chaotic stochastic resonance energy detection fusion used in cooperative spectrum sensing," IEEE Transactions on Vehicular Technology, vol. 62, no. 2, pp. 620-627, 2013.

[7] H. L. V. Trees, Detection, Estimation, and Modulation Theory, Optimum Array Processing, Wiley-Interscience, 2002.

[8] T. Okano, A. Kitagawa, and K. Miyakawa, "Array-enhanced coherence resonance and phase synchronization in a twodimensional array of excitable chemical oscillators," Physical Review E: Statistical, Nonlinear, and Soft Matter Physics, vol. 76, no. 4, Article ID 046201, 6 pages, 2007.

[9] Y. Tang, W. Zou, J. Lu, and J. Kurths, "Stochastic resonance in an ensemble of bistable systems under stable distribution noises and nonhomogeneous coupling," Physical Review E, vol. 85, no. 4, Article ID 046207, 6 pages, 2012.

[10] D. Rousseau and F. Chapeau-Blondeau, "Constructive role of noise in signal detection from parallel arrays of quantizers," Signal Processing, vol. 85, no. 3, pp. 571-580, 2005.

[11] F. Duan, F. Chapeau-Blondeau, and D. Abbott, "Noiseenhanced SNR gain in parallel array of bistable oscillators," Electronics Letters, vol. 42, no. 17, pp. 1008-1009, 2006.

[12] F. Duan, F. Chapeau-Blondeau, and D. Abbott, "Stochastic resonance in a parallel array of nonlinear dynamical elements," Physics Letters A, vol. 372, no. 13, pp. 2159-2166, 2008. 
[13] L. Gammaitoni, P. Hänggi, P. Jung, and F. Marchesoni, "Stochastic resonance," Reviews of Modern Physics, vol. 70, no. 1, pp. 223287, 1998.

[14] T. Wellens, V. Shatokhin, and A. Buchleitner, "Stochastic resonance," Reports on Progress in Physics, vol. 67, no. 1, pp. 45-105, 2004.

[15] B. McNamara and K. Wiesenfeld, "Theory of stochastic resonance," Physical Review A, vol. 39, no. 9, pp. 4854-4869, 1989.

[16] P. Jung, U. Behn, E. Pantazelou, and F. Moss, "Collective response in globally coupled bistable systems," Physical Review A, vol. 46, no. 4, pp. R1709-R1912, 1992.

[17] M. Locher, D. Cigna, E. R. Hunt et al., "Stochastic resonance in coupled nonlinear dynamic elements," Chaos, vol. 8, no. 3, pp. 604-615, 1998.

[18] M. Locher, N. Chatterjee, F. Marchesoni, W. L. Ditto, and E. R. Hunt, "Noise sustained propagation: local versus global noise," Physical Review E, vol. 61, no. 5, pp. 4954-4961, 2000.

[19] F. Duan, F. Chapeau-Blondeau, and D. Abbott, "Noiseenhanced snr gain in parallel array of bistable oscillators," Electronics Letters, vol. 42, no. 17, pp. 1008-1009, 2006.

[20] F. Duan, F. Chapeau-Blondeau, and D. Abbott, "Stochastic resonance in a parallel array of nonlinear dynamical elements," Physics Letters A, vol. 372, pp. 2159-2166, 2008.

[21] L. Gammaitoni, P. Hanggi, P. Jung, and F. Marchesoni, "Stochastic resonance," Reviews of Modern Physics, vol. 70, no. 1, pp. 223287, 1998.

[22] T. Wellens, V. Shatokhin, and A. Buchleitner, "Stochastic resonance," Reports on Progress in Physics, vol. 67, pp. 45-105, 2004.

[23] C. Nicolis, "Stochastic aspects of climatic transitions response to a periodic forcing," Tellus, vol. 34, no. 1, pp. 1-9, 1982.

[24] B. McNamara and K. Wiesenfeld, "Theory of stochastic resonance," Physical Review A, vol. 39, pp. 4854-4869, 1989. 


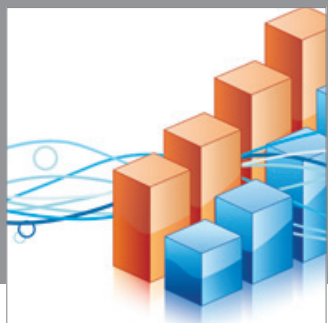

Advances in

Operations Research

mansans

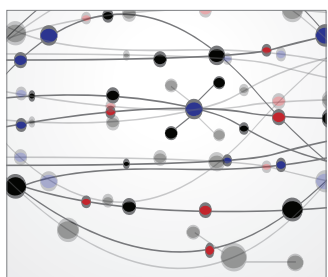

The Scientific World Journal
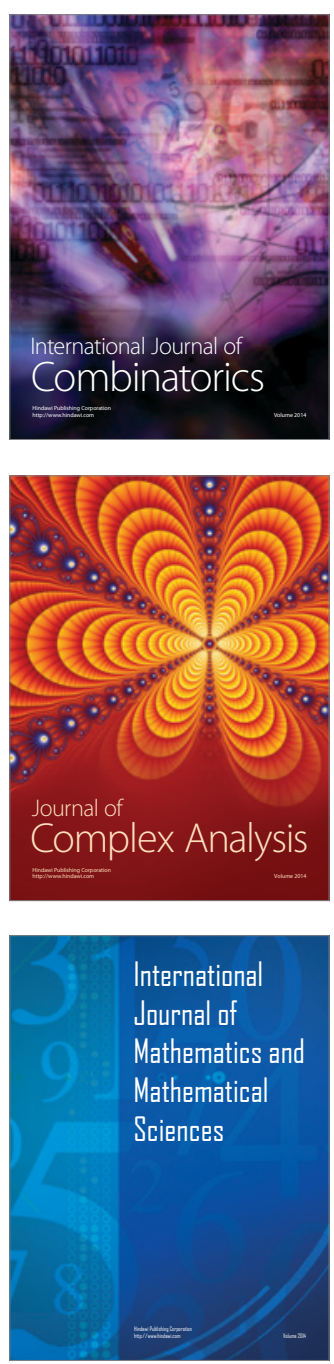
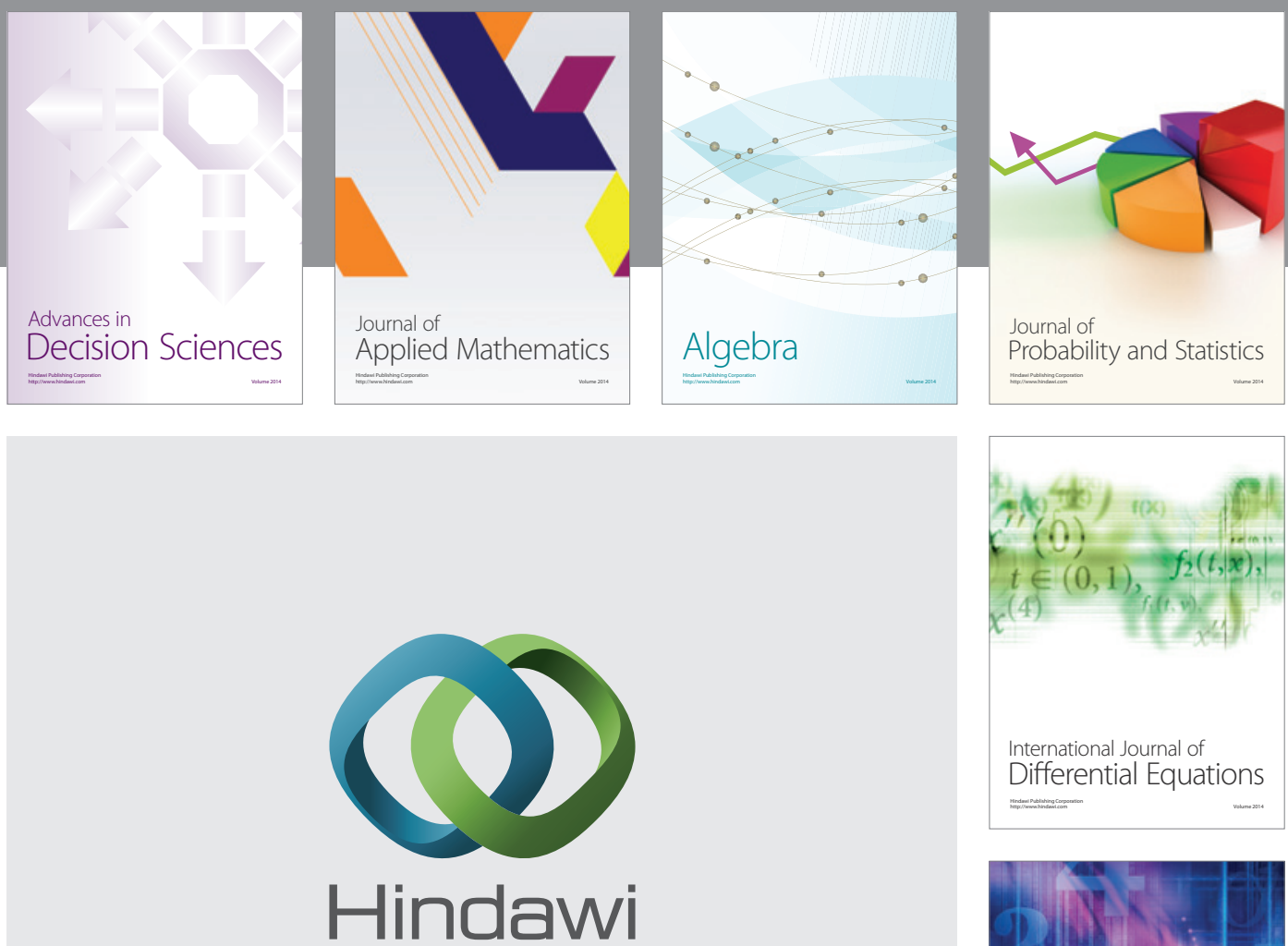

Submit your manuscripts at http://www.hindawi.com
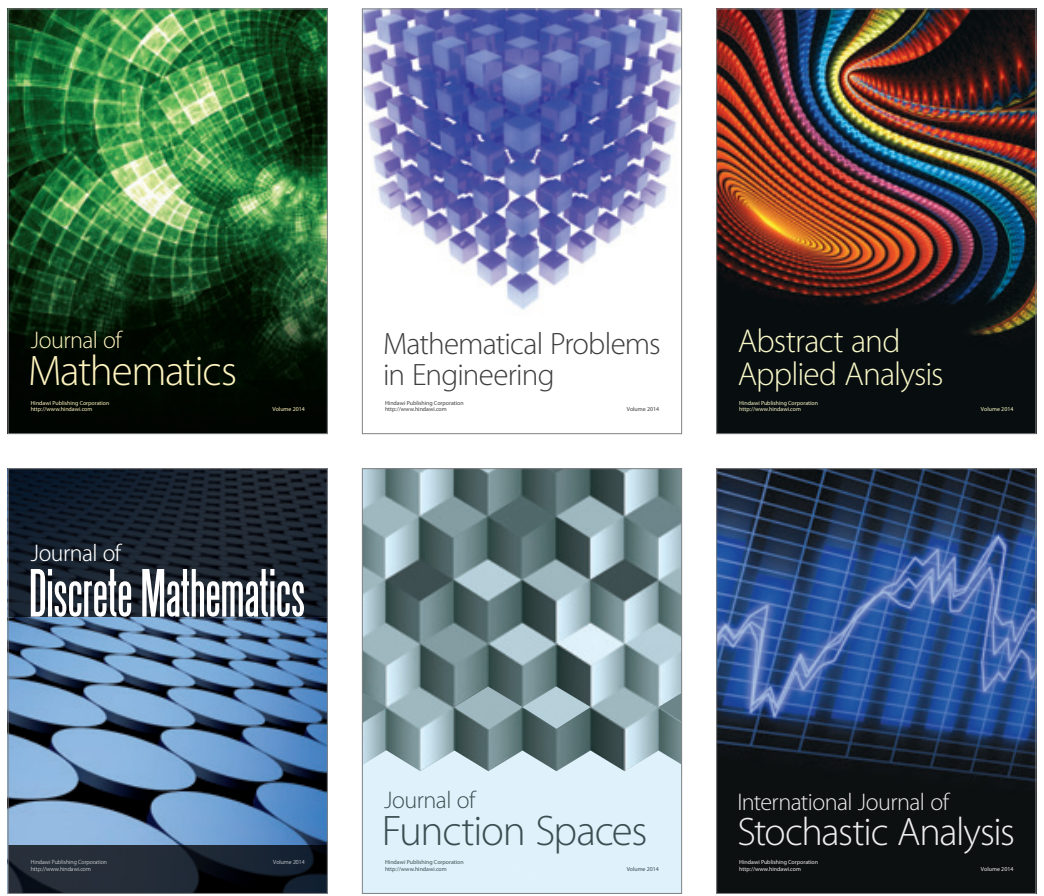

Journal of

Function Spaces

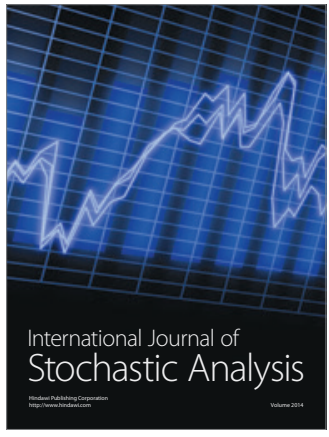

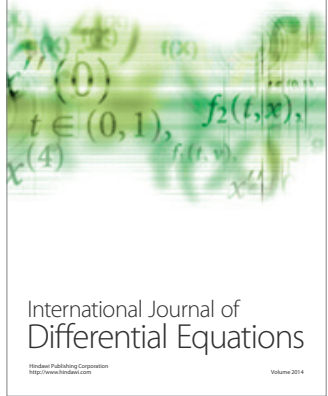
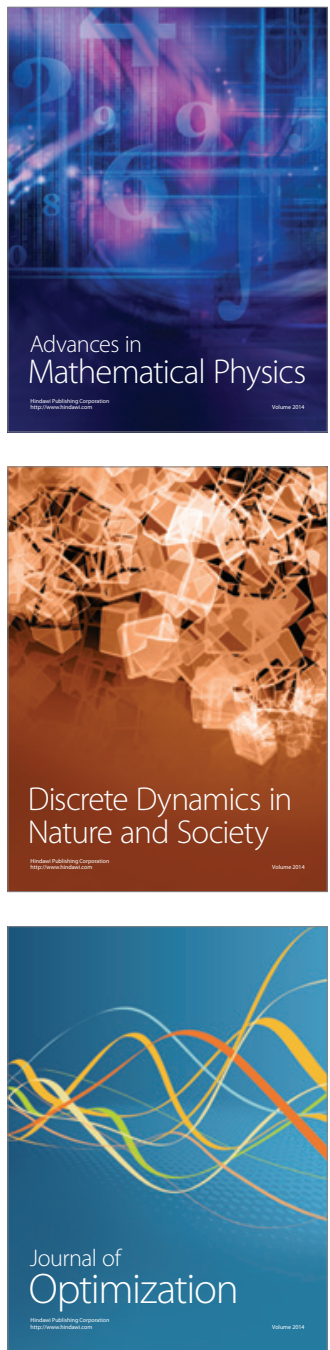